\title{
Editorial \\ The behavioural pharmacology of stress
}

Behavioural Pharmacology 2014, 25:337-339

Stress is recognized as a major factor in the etiology of psychiatric disorders, yet the interplay between stress and behaviour that contributes to such illnesses remains poorly understood. What determines the wide variety of presentations of stress-related disorders? How do environmental stressors interact with genetic and developmental factors? What are the critical features that determine the perceived intensity of stressors? To what extent are different stressors (e.g. social and physical) equivalent in their effects, and why? To what extent are the numerous animal models of stress interchangeable? These big questions do not even touch upon the underlying brain mechanisms. This Special Issue does not attempt to offer a comprehensive account of the behavioural pharmacology of stress or, indeed, to provide a complete answer to any of these questions, but the 11 reviews and 10 empirical papers included do present a wealth of ideas and data that offer direction and, in some cases, partial answers to many of them.

As a framework within which to locate many of the papers included in this Special Issue, we can consider that the effects of stress depend upon a set of interdependent variables: the perceived intensity of the stimuli that constitute the stressor, the appraisal of the significance of those stimuli (in relation to factors such as danger or controllability), the neuropsychological context within which the stressor is located (in relation to factors such as genetic polymorphisms and prior stress exposure), and individual differences in the responsiveness of relevant physiological systems such as the hypothalamus-pituitary-adrenal (HPA) axis, other neuroendocrine neurochemical, or neuroimmune systems, and the autonomic nervous system. In the first review paper of this Special Issue, Lewis and Olive discuss one set of mechanisms underlying the effects of early-life stress - which in later life can influence all of the variables listed above - epigenesis. Their wideranging review covers recent evidence on two commonly investigated epigenetic mechanisms, histone modifications and DNA methylation, which can alter patterns of gene expression, consequent changes in the adult phenotype, and the risk for psychiatric illness. The authors also examine epigenetic effects on a number of key neurochemical systems, epigenetic influences of early-life, prenatal and adult stress, and the exciting new area of transgenerational epigenetics, and offer an introduction to the prospects of epigenetic psycho- therapeutics. For anyone needing an introduction to this burgeoning literature, this paper is a must-read.

In the second review paper, Willner et al. provide an important new theoretical perspective on the treatment of depression. Episodes of depression are typically precipitated by stress, but the intensity of stress needed to precipitate depression may be very low in individuals whose vulnerability is heightened by factors such as a prior history of depression, childhood trauma, or genetic risk factors. It is now widely accepted that stress damages the hippocampus and antidepressants act primarily by repairing that damage. The main point of this paper is to demonstrate a relationship between susceptibility to depression (i.e. low levels of stress precipitation) and resistance to antidepressant treatment. The authors argue that this relationship implies that the prospects for improving antidepressant efficacy from within the current research paradigm are limited, and that both clinical and preclinical research should focus more on predispositional variables and less on the hippocampus. The one major exception to the rule that a high risk for depression is associated with a poor response to antidepressants is the sex difference; women are at a higher risk for depression, but overall, women and men respond similarly to antidepressant drugs, suggesting that the physiological basis of the sex difference in depression is different from that for other risk factors. This position is supported by studies reviewed by Francescelli $e t$ al. on sex differences in the best-validated animal model of depression, chronic mild stress (CMS). This is an underresearched area, but overall, unlike the situation in humans, there is little, if any, evidence that female rats are more at risk for depressive-like effects of stress. In a third depression-related review, Haleem evaluates the potential role of leptin - usually considered to be an antiobesity hormone - in stress controllability. This possibility arises because, in addition to its role as a negative feedback adiposity signal, circulating leptin can cross the blood-brain barrier and influence HPA activity. However, although there is some evidence that leptin may have antidepressant or anxiolytic effects, the clinical data are somewhat inconclusive.

Post-traumatic stress disorder (PTSD) is a second major stress-related psychiatric illness, which has proven resistant to drug treatment and is also difficult to model in animals because of the subjective nature of the major psychological symptom, intrusive re-experiencing of past 
trauma. Nevertheless, animal models of symptoms associated with PTSD have been developed, and these have been reviewed by Whitaker et al. Various models have been described, involving diverse physical, social, and psychological stressors, with particular focus on attempts to model the comorbidity between PTSD and alcohol use disorders. The review draws out, inter alia, neurobiological parallels with PTSD such as alterations in the functioning of the amygdala or prefrontal cortex in response to reminders of the traumatic stress. A complementary review by Sun and Alkon provides a detailed account of the neurobiology and neuropharmacology of PTSD and stress-related effects on cognition, including memory of traumatic events and stress-induced memory impairment. They suggest a number of ways in which stress-related cognitive impairments and biases might be counteracted pharmacologically, such as by enhancement of cognitive functions, repair of stress-induced brain damage, enhancement of fear extinction, or experimental amnesia for traumatic events. One neurobiological focus of this paper, the endocannabinoid system, is considered in greater depth by Batista $e t$ al., who review the profile of endocannabinoid reuptake inhibitors and hydrolysis inhibitors, which enhance endocannabinoid activity, in experimental tests predictive of anxiolytic activity. They conclude that these drugs may have anxiolytic, and perhaps also antidepressant, activity without the abuserelated effects of exocannabinoids, while cautioning, however, against overoptimism.

The next two reviews address aspects of stress in relation to addiction. Fields et al. have carried out a meta-analysis of the clinical literature investigating the relationship between stress and impulsivity, as assessed using delay discounting procedures, which evaluate the preference for immediate over delayed outcomes. Impulsivity has been related to a range of psychiatric disorders, including abuse of a variety of substances, aggression, and eating disorders. The results of the meta-analysis confirm that higher levels of stress are associated with significantly increased impulsivity, with a larger effect in adults than in children, which contributes to an explanation of how stress can increase certain forms of psychiatric morbidity. Bisagno and Cadet review sex differences in addiction, which, unlike the situation for depression, appear to be relatively consistent between humans and animal models. They go on to discuss the sexually dimorphic involvement of neurochemical systems in these effects, with particular reference to two stress-responsive peptides, corticotropin-releasing factor (CRF) and oxytocin/vasopressin.

The final two reviews present the case that certain drug experiences should considered as stressors. Parrott et al. review the evidence that 3,4-methylenedioxymethamphetamine (MDMA, ecstasy) increases stress levels in humans by stimulating the HPA axis, and that this effect is greatly magnified when ecstasy/MDMA is used while dancing; hence, explaining why recreational ecstasy/MDMA users show symptoms typically associated with high levels of stress. Chartoff and Carlezon argue that withdrawal from drugs of abuse is a stress-like state, on the basis of extensive parallels between drug withdrawal and conventional stressors in relation to behavioural, neurochemical, and molecular endpoints. These parallels include the fact that, although both situations are typically aversive, there are circumstances under which both stress and drug withdrawal may act as positive reinforcers. They suggest that a better understanding of this relationship could improve the treatment of both addiction and other stress-related disorders.

As in the review section of this Special Issue, the Research Reports also begin with a study of an important predispositional factor determining responses to stress, personality. Childs et al. measured physiological responses to a social stressor (public speaking) in healthy volunteers and found that several personality traits, relating to neuroticism, social dominance, and affiliative motivation, each predicted different profiles of emotional, cardiovascular, and cortisol responses. The mechanisms underlying these differences will be of interest to determine, as will the implications for differential health risks.

Continuing the individual differences theme, Nesic and Duka, using a similar social stress procedure, asked whether stress would differentially affect alcohol craving in human binge drinkers compared with nonbinge drinkers with similar overall levels of alcohol consumption. They found that stress did indeed increase craving to a greater degree in binge drinkers (and also that this effect of stress was moderated differently in bingers and nonbingers by a dietary enhancement of 5-hydroxytryptamine function). One important difference between bingers and nonbingers is that during the intervals between binges, bingers experience alcohol withdrawal. Papp et al. report similar withdrawal data in rats. Animals were exposed chronically to CMS then, while still under stress, were subchronically exposed to three very different drugs of abuse: morphine, nicotine, and diazepam. CMS exacerbated both precipitated and cued withdrawal effects, as well as withdrawal-associated and cue-associated place aversions following withdrawal from all three drugs. All of these effects were reversed by chronic administration of the selective serotonin reuptake inhibitor citalopram, leading to the suggestion that treatment of comorbid depression might facilitate other treatments for addiction, by decreasing the severity of withdrawal symptoms and, thus, the likelihood of relapse. The pervasive aversive effects of stress are also illustrated by the demonstration by Garcia-Pardo et al. that, in common with other psychostimulant drugs, the rewarding effect of MDMA, as assessed using place-preference conditioning, is decreased by repeated social defeat stress. These dual effects of stress - that is, to increase 
aversion and decrease reward - are, of course, highly relevant to depression.

Two further papers concern specific neurochemical adaptations to stress. Considering that the endocannabinoid system has been implicated in a range of psychiatric disorders, early-life stress is known to increase risk, and many psychiatric disorders first present during adolescence, Marco et al. studied the expression of a comprehensive set of genes related to the endocannabinoid system in adolescent rats following the early-life stress of maternal deprivation. They report major perturbations of endocannabinoid expression in the adolescent brain following early-life stress, and that the effects are sexually dimorphic, which may be relevant to understanding sex differences in the expression of psychiatric symptomatology. Matuszewich et al. highlight the sometimes unpredictable relationship between stress and its effects on neurochemical and performance endpoints. They report that chronic unpredictable stress improved performance on a spatial recognition task while decreasing dopamine release in the prefrontal cortex following exposure to a mild acute stressor, which is inconsistent with an earlier report that CMS has the opposite effect on prefrontal dopamine release and the typical finding that CMS causes cognitive impairments. These findings emphasize the often overlooked yet critical fact that minor differences in stress procedures may have major behavioural consequences.

The next three papers in this Special Issue report the results of attempts to reverse depression-related stress effects pharmacologically. Another paper by Papp et al. reports that cariprazine, a dopamine D2/D3 partial agonist that binds preferentially to D3 receptors, shows an antidepressant-like reversal of anhedonia in the rat CMS model. They suggest that cariprazine might have clinical utility not only in the treatment of depression, but also, given the dopaminergic mechanism of action, in relation to the negative symptoms of schizophrenia. Kanno et al. report that a mixture of glycerol-3-phosphocholine derivatives restored depression-related behaviours and spatial memory impairment in rats following repeated restraint stress, possibly by inhibiting glycogen synthase kinase $3 \beta$, an important element in post-transductional 5-hydroxytryptamine signaling that has been implicated in depression and dementia, as well as in the antidepressant action of lithium. In the same spirit, Cantanzaro et al. took as their starting point the observation that the $\mathrm{P}_{2} \mathrm{X}_{7}$ receptor plays an important role in the synthesis of the inflammatory cytokine inerleukin-1 $\beta$ which, in turn, has been proposed to play an important role in the response to stress. In a series of experiments a $\mathrm{P}_{2} \mathrm{X}_{7}$ receptor antagonist was administered to rats subjected to footshock, with the presumption that markers for stress would be reduced. However, the behavioural and neurochemical effects reported were somewhat inconclusive, providing a refreshing accounting of a valuable series of experiments that failed to support an otherwise attractive hypothesis.

In the final paper, Hymel et al. tested the hypothesis that amnestic agents might have utility in the (notoriously difficult) treatment of PTSD. Mice were subjected to repeated social defeat stress and showed characteristic stress-related behavioural changes. They were then subjected to a single further exposure to social defeat while under the influence of propranolol or cycloheximide, drugs known to disrupt memory consolidation, which caused a dose-dependent normalization of behavioural responses tested a day later. The results suggest that disruption of the reconsolidation of a labile traumatic memory reactivated by re-exposure to stress could be used in the treatment of established PTSD symptoms an exciting prospect.

In sum, the wide range of reviews and reports in this Special Issue reflect the many streams of research that flow into our current understanding of stress, its role in psychiatric disorders, and how to combat it effectively. As emphasized in the opening paragraph, this current understanding is woefully incomplete, which handicaps our approaches to treatment. Yet, it is equally important to emphasize that the many approaches touched upon in this Special Issue, and others, will inevitably lead us to a better appreciation of how stressful factors in daily life impact both normative and maladaptive behaviours.

Paul Willner Jack Bergman Louk Vanderschuren and Bart Ellenbroek July 2014 